\title{
Hot QCD at finite isospin density: Confronting the SU(3) Nambu-Jona-Lasinio model with recent lattice data
}

\author{
Bruno S. Lopes $\odot,{ }^{1}$ Sidney S. Avancini®, ${ }^{2}$ Aritra Bandyopadhyay $\odot,{ }^{3,4}$ \\ Dyana C. Duarte $\odot,{ }^{5,6}$ and Ricardo L. S. Farias $\oplus^{1}$ \\ ${ }^{1}$ Departamento de Física, Universidade Federal de Santa Maria, \\ Santa Maria, Rio Grande do Sul 97105-900, Brazil \\ ${ }^{2}$ Departamento de Física, Universidade Federal de Santa Catarina, \\ 88040-900 Florianópolis, Santa Catarina, Brazil \\ ${ }^{3}$ Guangdong Provincial Key Laboratory of Nuclear Science, Institute of Quantum Matter, South China \\ Normal University, Guangzhou 510006, China \\ ${ }^{4}$ Guangdong-Hong Kong Joint Laboratory of Quantum Matter, Southern Nuclear Science Computing \\ Center, South China Normal University, Guangzhou 510006, China \\ ${ }^{5}$ Institute for Nuclear Theory, University of Washington, Seattle, Washington 98195, USA \\ ${ }^{6}$ Departamento de Física, Instituto Tecnológico de Aeronáutica, \\ 12228-900 São José dos Campos, São Paulo, Brazil
}

(Received 9 February 2021; accepted 1 April 2021; published 30 April 2021)

\begin{abstract}
Extending our recently published SU(2) results for zero temperature, we now compute the QCD equation of state for finite isospin density within the three-flavor Nambu-Jona-Lasinio (NJL) model in the mean field approximation, motivated by the recently obtained lattice QCD results for both zero and finite temperatures. Like our previous study, here also we have considered both the commonly used traditional cutoff regularization scheme and the medium separation scheme. Our results are compared with recent high-precision lattice simulations as well as previously obtained results in two-flavor Nambu-Jona-Lasinio model. The agreement between the lattice results and the predictions from three-flavor NJL model is very good for low values of $\mu_{I}$ (for both zero and finite temperatures). For larger values of $\mu_{I}$, the agreement between lattice data and the two-flavor NJL predictions is surprisingly good and better than with the threeflavor predictions.
\end{abstract}

DOI: $10.1103 /$ PhysRevD.103.076023

\section{INTRODUCTION}

As the fundamental theory of strong interactions, the phase structure of quantum chromodynamics (QCD) has been studied from different angles over the years. Aside from the well-explored systems at finite temperatures and finite baryon densities, several new dimensions have been added to the QCD phase diagrams: isospin chemical potential, magnetic field, electric field, and rotation to name a few. Though near future relativistic heavy-ion-collision experiments in Facility for Antiproton and Ion Research (FAIR) and Nuclotron-based Ion Collider Facility (NICA) have been continuing to inspire studies of physical systems at low temperatures and finite baryon densities such as neutron stars $[1,2]$, theoretical hurdles are still there, predominantly due to the well-known fermion "sign problem" [3,4] encountered

Published by the American Physical Society under the terms of the Creative Commons Attribution 4.0 International license. Further distribution of this work must maintain attribution to the author(s) and the published article's title, journal citation, and DOI. Funded by SCOAP ${ }^{3}$. by nonperturbative lattice calculations. Lattice QCD's recent progress with the sign problem can be monitored in Ref. [5].

Among the relatively new features of the QCD phase diagram, finite isospin chemical potential $\left(\mu_{I}\right)$ plays an important role, specially because unlike finite baryon chemical potential it does not suffer from the sign problem in lattice QCD-based calculations. First bunch of lattice QCD results at finite temperature and isospin density appeared in early 2000s [6,7] with dynamical $u$ and $d$ quarks, although with unphysical pion masses and/or an unphysical flavor content. This followed various studies by other available theoretical tools yielding qualitatively similar results. These studies include chiral perturbation theory $(\chi \mathrm{PT})[8-20]$, hard thermal loop perturbation theory [21], Nambu-Jona-Lasinio (NJL) model [22-42] and its Polyakov loop extended version PNJL $[43,44]$, and quark meson model [45-48]. Recently, early lattice QCD results have been modified by using an improved lattice action with staggered fermions at physical quark masses and results for finite isospin density are presented in Refs. [49-52]. 
In our recent work along the similar line [53], we focused on a new type of compact stars known as pion stars [11,54], where the pion condensates are considered to be the dominant constituents of the core under the circumstance of vanishing neutron density. In spite of being a subset of boson stars [55-59], pion stars are free from hypothetical beyond standard model contributions like QCD axion. This gave us a scenario to work with finite isospin density along with zero temperature and zero baryon density which bypasses the sign problem unlike systems with high baryon densities. Hence, it is easily accessible through first principle methods [54] and through the pion stars' equation of state we now know about its large mass and radius in comparison with neutron stars $[54,60]$. Our study in the said context of pion stars within two-flavor NJL model showed better quantitative agreement with the lattice QCD results than similar studies within the chiral perturbation theory [18].

Unlike our last study [53], where we have only considered the setting of pion stars with zero temperature, in the present work we plan also to explore the systems with finite temperature. Early universe with very high temperature has been known to have possibilities of pion condensation driven by high lepton asymmetry [61-63]. Furthermore, in this work, we have extended our two-flavor study within the three-flavor NJL model. While the two-flavor studies are sufficient to describe the pion condensation at finite isospin density, a three-flavor study gives us the chance to explore the roles of the strangeness degree of freedom and the $U_{A}(1)$ anomaly in the present context. Hence, in our three- flavor NJL Lagrangian, we will also be considering the Kobayashi-Maskawa-'t Hooft (KMT) term, which mimics the $U_{A}(1)$ anomaly in the NJL model.

Just like QCD systems with $\mu_{I} \neq 0, \mu_{B}=\mu_{s}=T=0$ [54], QCD systems with $\mu_{I} \neq 0, \mu_{B}=0, \mu_{s} \neq 0, T \neq 0$ are also being explored well within lattice QCD [49,52,64-66]. Successful premises of this work have already been provided by our previous study [53], which showed an exceptional quantitative agreement between NJL and lattice QCD results. On the basis of that and recently improved three-flavor lattice QCD, results at zero and finite temperature $[49,52,54,64-66]$ give us the perfect opportunity for the consistency check of the NJL model. As in our previous work [53], we have tried to rectify the regularization issues within NJL model to deal with the cutting of important degrees of freedom near the Fermi surface because of a sharp ultraviolet cutoff [67-69]. Besides the commonly used traditional regularization scheme (TRS), we have used the medium separation scheme (MSS) [67,70,71], which properly separates the medium effects from divergent integrals. For systems with high values of $\mu_{I}(\sim \Lambda)$, the role of MSS becomes more and more important.

The paper is organized as follows. In Sec. II, we discuss the basic formalism of the three-flavor NJL model both within TRS and MSS. In Sec. III, we present and discuss our results obtained with the traditional regularization scheme and with the medium separation scheme, for both zero and finite temperature. Thermodynamic results and the $T-\mu_{I}$ phase diagram are also presented and compared with the other state-of-the-art calculations.

\section{FORMALISM}

We start with the partition function for the three-flavor NJL model at finite baryonic and isospin chemical potential, given by

$$
\begin{aligned}
& Z_{\mathrm{NJL}}\left(T, \mu_{B}, \mu_{I}, \mu_{S}\right) \\
& \quad=\int[d \bar{\psi}][d \psi] \times \exp \left[\int_{0}^{\beta} d \tau \int d^{3} x\left(\mathcal{L}_{\mathrm{NJL}}+\bar{\psi} \hat{\mu} \gamma_{0} \psi\right)\right],
\end{aligned}
$$

where the quark chemical potential matrix in flavor space is

$$
\hat{\mu}=\left(\begin{array}{ccc}
\mu_{u} & 0 & 0 \\
0 & \mu_{d} & 0 \\
0 & 0 & \mu_{s}
\end{array}\right),
$$

and $\mu_{u, d, s}$ can be expressed in terms of the baryonic, the isospin, and the strangeness chemical potential as

$$
\begin{aligned}
& \mu_{u}=\frac{\mu_{B}}{3}+\frac{\mu_{I}}{2}, \\
& \mu_{d}=\frac{\mu_{B}}{3}-\frac{\mu_{I}}{2}, \\
& \mu_{s}=\frac{\mu_{B}}{3}-\mu_{S},
\end{aligned}
$$

such that $\mu_{I}=\left(\mu_{u}-\mu_{d}\right) .{ }^{1} \mathcal{L}_{\mathrm{NJL}}$ appearing in Eq. (2.1) is the NJL Lagrangian considering scalar and pseudoscalar interactions, i.e.,

$$
\begin{aligned}
\mathcal{L}_{\mathrm{NJL}}= & \bar{\psi}(i \not \supset-m) \psi+G \sum_{\alpha=0}^{N_{f}^{2}-1}\left[\left(\bar{\psi} \lambda_{\alpha} \psi\right)^{2}+\left(\bar{\psi} i \gamma_{5} \lambda_{\alpha} \psi\right)^{2}\right] \\
& -K\left[\operatorname{det} \bar{\psi}\left(1+\gamma_{5}\right) \psi+\operatorname{det} \bar{\psi}\left(1-\gamma_{5}\right) \psi\right],
\end{aligned}
$$

where $\psi=(u d s)^{T}$ and $m=\operatorname{diag}\left(m_{u}, m_{d}, m_{s}\right)$ represent the quark fields and their current mass, respectively, and $G$ is the scalar coupling constant of the model from the fourfermion interaction. The last term is the KMT term which represents the breaking of the flavor symmetry in the chiral limit due to $U_{A}(1)$ anomaly. $K$ is also known as the $U_{A}(1)$ breaking strength.

\footnotetext{
${ }^{1}$ We would like to point out here that Ref. [53] has typos in the definitions of $\mu_{u / d}$ and in the dispersion relation, where $\mu_{I}$ should be replaced by $\mu_{I} / 2$.
} 
Next, in the mean field approximation, we introduce the chiral condensates

$\sigma_{l}=\sigma_{u / d}=-4 G\langle\bar{u} u\rangle /\langle\bar{d} d\rangle, \quad \sigma_{s}=-4 G\langle\bar{s} s\rangle$

and the pseudoscalar pion condensate

$$
\Delta=2\left(2 G-K \sigma_{s}\right)\left\langle\bar{u} i \gamma_{5} d\right\rangle,
$$

where $\Delta$ can be considered as real without loss of generality as the related phase factor can be arbitrarily chosen due to the spontaneously broken $U_{I}(1)$ symmetry $^{2}$ [29]. In terms of these condensates, the thermodynamic potential for $N_{f}=2+1$ in the mean field approximation is given as

$$
\begin{aligned}
\Omega\left(\sigma_{l}, \sigma_{s}, \Delta\right)= & \frac{2 \sigma_{l}^{2}+\sigma_{s}^{2}}{8 G}+\frac{K \sigma_{s} \sigma_{l}^{2}}{16 G^{3}} \\
& +\left(G+\frac{K \sigma_{s}}{4 G}\right) \times \frac{\Delta^{2}}{\left(2 G+\frac{K \sigma_{s}}{4 G}\right)^{2}} \\
& -2 N_{c} \int_{k}^{\Lambda}\left[E_{k}^{+}+E_{k}^{-}+E_{k}^{s}+2 T\right. \\
& \left.\times \ln \left\{\left(1+e^{-\beta E_{k}^{-}}\right)\left(1+e^{-\beta E_{k}^{+}}\right)\left(1+e^{-\beta E_{k}^{s}}\right)\right\}\right],
\end{aligned}
$$

with $E_{k}^{ \pm}=\sqrt{\left(E_{k}^{l} \pm \frac{\mu_{I}}{2}\right)^{2}+\Delta^{2}}$ with $E_{k}^{l}=\sqrt{k^{2}+M_{l}^{2}}$ and $E_{k}^{s}=\sqrt{k^{2}+M_{s}^{2}}$ and the symbol $\int_{k}^{\Lambda}$ indicates three momentum integrals that need to be regularized. Different effective masses $M_{l}$ and $M_{s}$ are defined as

$$
\begin{gathered}
M_{l}=m_{l}+\sigma_{l}+\frac{K \sigma_{l} \sigma_{s}}{8 G^{2}}, \\
M_{s}=m_{s}+\sigma_{s}+\frac{K \sigma_{l}^{2}}{8 G^{2}}+\frac{K}{2} \frac{\Delta^{2}}{\left(2 G+\frac{K \sigma_{s}}{4 G}\right)^{2}},
\end{gathered}
$$

where $m_{l}=m_{u}=m_{d}$ and $m_{s}$ are the current quark masses.

The ground state at finite temperature and isospin chemical potential is determined by minimizing $\Omega\left(\sigma_{l}, \sigma_{s}, \Delta\right)$ with respect to $\sigma_{l}, \sigma_{s}$, and $\Delta$, i.e., by solving $\partial \Omega / \partial \sigma_{l}=$ $\partial \Omega / \partial \sigma_{s}=\partial \Omega / \partial \Delta=0$.

In the following subsections, we discuss in more details different ways of regularizing these integrals. The thermodynamic quantities, i.e., the pressure, the isospin density, and the energy density of the system are then, respectively, given by

$$
P_{\mathrm{NJL}}=-\Omega_{\mathrm{NJL}}\left(\sigma_{l / s}=\sigma_{l / s}^{0} ; \Delta=\Delta^{0}\right),
$$

\footnotetext{
${ }^{2}$ It is important to note here that in this work we are not considering kaon condensation as we are working in the limit of vanishing baryonic and strangeness chemical potentials, i.e., $\mu_{B}=\mu_{S}=0$.
}

$$
\begin{gathered}
\left\langle n_{I}\right\rangle_{\mathrm{NJL}}=\frac{\partial P_{\mathrm{NJL}}}{\partial \mu_{I}} \\
\varepsilon_{\mathrm{NJL}}=-P_{\mathrm{NJL}}+\mu_{I}\left\langle n_{I}\right\rangle_{\mathrm{NJL}}+T \frac{\partial P_{\mathrm{NJL}}}{\partial T} .
\end{gathered}
$$

Finally, the interaction measure (or trace anomaly) within the NJL model is given by the relation between $P_{\mathrm{NJL}}$ and $\varepsilon_{\mathrm{NJL}}$, i.e.,

$$
I_{\mathrm{NJL}}=\varepsilon_{\mathrm{NJL}}-3 P_{\mathrm{NJL}}
$$

\section{A. Regularization}

Due to the nonrenormalizable nature of the NJL model, any physical quantity determined in its framework will be dependent on the scale of the model $\Lambda$. In the SU(2) version, the usual regularization schemes consist in to determine $\Lambda$, the coupling constant $G$, and current quark mass $m_{u}=m_{d}=m_{c}$ that reproduce the empirical values of the pion mass $m_{\pi}$, the pion decay constant $f_{\pi}$, and the quark condensate $\langle\bar{\psi} \psi\rangle\rangle$. Since our aim is to compare our results with lattice simulations, we have used two different sets of parameters, for $T=0$ and $T \neq 0$, as can be seen in Table I.

The $\mathrm{SU}(3)$ case is much more complicated; the procedure is shown in details in the Appendix and the values obtained are shown in Table II. The two sets represent the parameters we use for two different cases, set I for the case of zero temperature and set II for the case of finite temperature, following the value of $m_{\pi}$ used by lattice QCD, respectively, for both the cases.

The fact that all physical quantities are dependent on $\Lambda$ does not mean that we can just naively use this cutoff in all integrals, since it may lead to incorrect results. In this work, we compare the results of two different schemes, namely, the TRS and the MSS. The TRS is the most common in NJL studies and consists only in to perform up to $\Lambda$ the integrals that do not depend on the temperature, e.g., the first three terms between brackets of the integral in Eq. (2.9) and its correspondents in the gap equations and thermodynamic quantities, while thermal integrals are performed up to infinity.

TABLE I. Different parameter sets are listed, which have been used in the present study for $\mathrm{SU}(2)$ case.

\begin{tabular}{lcc}
\hline \hline Sets & Input parameters & Output parameters \\
\hline I & $f_{\pi}=93 \mathrm{MeV}$ & $\Lambda=659.325 \mathrm{MeV}$ \\
& $m_{\pi}=131.7 \mathrm{MeV}$ & $G=2.07835 / \Lambda^{2}$ \\
& $\langle\bar{\psi} \psi\rangle^{1 / 3}=250 \mathrm{MeV}$ & $m_{c}=4.757 \mathrm{MeV}$ \\
II & $f_{\pi}=92.4 \mathrm{MeV}$ & $\Lambda=659.325 \mathrm{MeV}$ \\
& $m_{\pi}=135.5263 \mathrm{MeV}$ & $G=2.07691 / \Lambda^{2}$ \\
& $\langle\bar{\psi} \psi\rangle^{1 / 3}=250 \mathrm{MeV}$ & $m_{c}=4.93651 \mathrm{MeV}$ \\
\hline \hline
\end{tabular}


TABLE II. Different parameter sets are listed, which have been used in the present study for SU(3) case.

\begin{tabular}{lcc}
\hline \hline Sets & Input parameters & Output parameters \\
\hline I & $f_{\pi}=93 \mathrm{MeV}$ & $\Lambda=574.68 \mathrm{MeV}$ \\
& $m_{\pi}=131.7 \mathrm{MeV}$ & $G=2.2066 / \Lambda^{2}$ \\
& $m_{K}=490 \mathrm{MeV}$ & $K=10.426 / \Lambda^{5}$ \\
$m_{\eta}=950 \mathrm{MeV}$ & $m_{s}=140 \mathrm{MeV}$ \\
& $m_{l}=5.3 \mathrm{MeV}$ & \\
& $f_{\pi}=92.4 \mathrm{MeV}$ & $\Lambda=608.431 \mathrm{MeV}$ \\
$\mathrm{II}$ & $m_{\pi}=135.5263 \mathrm{MeV}$ & $G=1.782 / \Lambda^{2}$ \\
& $m_{K}=497.7 \mathrm{MeV}$ & $K=12.8525 / \Lambda^{5}$ \\
& $m_{\eta}=957.8 \mathrm{MeV}$ & $m_{s}=140.305 \mathrm{MeV}$ \\
& $m_{l}=5.5 \mathrm{MeV}$ & \\
\hline \hline
\end{tabular}

On the other hand, MSS provides a tool to disentangle all the medium dependencies from divergent contributions, so that only vacuum integrals remain to be regularized. This scheme has been applied to the NJL model and successfully shows qualitative agreement with lattice simulations and more elaborated theories, as might be seen in Refs. $[53,67,70,71]$. Let us start, e.g., from integral $I_{\Delta}$ of $\Delta$ gap equation

$$
I_{\Delta}=\sum_{j= \pm 1} \int_{\Lambda} \frac{d^{3} k}{(2 \pi)^{3}} \frac{1}{\sqrt{\left(E_{k}^{l}+j \mu\right)^{2}+\Delta^{2}}}
$$

whose TRS version is obtained just by making the replacement $\int_{\Lambda} \frac{d^{3} k}{(2 \pi)^{3}} \rightarrow \int_{0}^{\Lambda} d k \frac{k^{2}}{2 \pi^{2}}$. To use MSS, we first rewrite

$I_{\Delta}=\frac{1}{\pi} \sum_{j= \pm 1} \int_{-\infty}^{+\infty} d x \int_{\Lambda} \frac{d^{3} k}{(2 \pi)^{3}} \frac{1}{x^{2}+\left(E_{k}^{l}+j \mu\right)^{2}+\Delta^{2}}$,

where, to ease the notation, we made the replacement $\mu_{I} / 2 \rightarrow \mu$. After two iterations of the following identity

$$
\begin{aligned}
& \frac{1}{x^{2}+\left(E_{k}^{l}+j \mu\right)^{2}+\Delta^{2}} \\
& =\frac{1}{x^{2}+k^{2}+M_{0}^{2}} \\
& +\frac{M_{0}^{2}-\Delta^{2}-\mu^{2}-M^{2}-2 j \mu E_{k}^{l}}{\left(x^{2}+k^{2}+M_{0}^{2}\right)\left[x^{2}+\left(E_{k}^{l}+j \mu\right)^{2}+\Delta^{2}\right]},
\end{aligned}
$$

where $M_{0}$ is the vacuum mass of light quarks, obtained in the $T=\mu=\Delta=0$ limit, we obtain

$$
\begin{aligned}
\sum_{j= \pm 1} & \frac{1}{x^{2}+\left(E_{k}^{l}+j \mu\right)^{2}+\Delta^{2}} \\
= & \frac{2}{x^{2}+k^{2}+M_{0}^{2}}+\frac{2 \mathcal{M}}{\left(x^{2}+k^{2}+M_{0}^{2}\right)^{2}} \\
& +\frac{2 \mathcal{M}^{2}+8 \mu^{2}\left(E_{k}^{l}\right)^{2}}{\left(x^{2}+k^{2}+M_{0}^{2}\right)^{3}} \\
& +\sum_{j= \pm 1} \frac{\left(\mathcal{M}-2 j \mu E_{k}^{l}\right)^{3}}{\left(x^{2}+k^{2}+M_{0}^{2}\right)^{3}\left[x^{2}+\left(E_{k}^{l}+j \mu\right)^{2}+\Delta^{2}\right]},
\end{aligned}
$$

where we have defined $\mathcal{M}=M_{0}^{2}-\Delta^{2}-\mu^{2}-M_{l}^{2}$. After some manipulations and performing the integration in $x$ indicated in (2.17), we obtain

$$
\begin{aligned}
I_{\Delta}^{\mathrm{MSS}}= & 2 I_{\mathrm{quad}}\left(M_{0}\right)-\left(M_{l}^{2}-M_{0}^{2}+\Delta^{2}-2 \mu^{2}\right) I_{\log }\left(M_{0}\right) \\
& +\left[\frac{3\left(\mathcal{M}^{2}+4 \mu^{2} M_{l}^{2}\right)}{4}-3 \mu^{2} M_{0}^{2}\right] I_{1}+2 I_{2}
\end{aligned}
$$

with the definitions

$$
\begin{gathered}
I_{\text {quad }}\left(M_{0}\right)=\int \frac{d^{3} k}{(2 \pi)^{3}} \frac{1}{\sqrt{k^{2}+M_{0}^{2}}}, \\
I_{\log }\left(M_{0}\right)=\int \frac{d^{3} k}{(2 \pi)^{3}} \frac{1}{\left(k^{2}+M_{0}^{2}\right)^{\frac{3}{2}}}, \\
I_{1}=\int \frac{d^{3} k}{(2 \pi)^{3}} \frac{1}{\left(k^{2}+M_{0}^{2}\right)^{\frac{5}{2}}}, \\
I_{2}=\frac{15}{32} \sum_{j= \pm 1} \int \frac{d^{3} k}{(2 \pi)^{3}} \int_{0}^{1} d t(1-t)^{2} \\
\times \frac{\left(\mathcal{M}-2 j \mu E_{k}^{l}\right)^{3}}{\left[\left(2 j \mu E_{k}^{l}-\mathcal{M}\right) t+k^{2}+M_{0}^{2}\right]^{\frac{7}{2}}} .
\end{gathered}
$$

A similar procedure can be used to obtain the integrals of other quantities,

$$
\begin{gathered}
I_{\sigma_{l}}=\sum_{j= \pm 1} \int_{\Lambda} \frac{d^{3} k}{(2 \pi)^{3}} \frac{1}{E_{k}^{l}} \frac{E_{k}^{l}+j \mu}{\sqrt{\left(E_{k}^{l}+j \mu\right)^{2}+\Delta^{2}}}, \\
I_{n_{I}}=\sum_{j= \pm 1} \int_{\Lambda} \frac{d^{3} k}{(2 \pi)^{3}} j \frac{E_{k}^{l}+j \mu}{\sqrt{\left(E_{k}^{l}+j \mu\right)^{2}+\Delta^{2}}}, \\
I_{\sigma_{s}}=\int_{\Lambda} \frac{d^{3} k}{(2 \pi)^{3}} \frac{1}{E_{k}^{s}} .
\end{gathered}
$$


Since the steps to obtain these integrals for MSS are described in detail in previous references [53,70,71], here we will just show the final results of each of these integrals,

$$
\begin{aligned}
I_{\sigma_{l}}^{\mathrm{MSS}}= & 2 I_{\text {quad }}\left(M_{0}\right)-\left(M_{l}^{2}-M_{0}^{2}+\Delta^{2}\right) I_{\log }\left(M_{0}\right)+I_{3} \\
& +3\left[\frac{\mathcal{M}^{2}}{4}+\mu^{2}\left(M_{l}^{2}-M_{0}^{2}-\mathcal{M}\right)\right] I_{1}+2 I_{2}, \quad(2.28) \\
I_{n_{I}}^{\mathrm{MSS}}= & 2 \mu \Delta^{2} I_{\log }\left(M_{0}\right) \\
& \times 3 \mu\left[\frac{\mathcal{M}^{2}}{4}+\mathcal{M}\left(M_{0}^{2}-M_{l}^{2}\right)+M_{l}^{2} \mu^{2}+\frac{2 \mu^{2} M_{0}^{2}}{3}\right] I_{1} \\
& +2 \mu I_{2}-\frac{5 \mu M_{l}^{2}}{4}\left[3 \mathcal{M}^{2}+4 \mu^{2} M_{l}^{2}\right] I_{4} \\
& +\frac{5 \mu}{4}\left(4 \mu^{2}\left(M_{0}^{2}-2 M_{l}^{2}\right)-3 \mathcal{M}^{2}\right) I_{5}+I_{6}, \\
I_{\sigma_{s}}^{\mathrm{MSS}}= & I_{\text {quad }}\left(M_{0 s}\right)+\frac{M_{0 s}^{2}-M_{s}^{2}}{2} I_{\log }\left(M_{0 s}\right)+I_{7},
\end{aligned}
$$

where $M_{0 s}$ is the vacuum strange quark mass, obtained in the $T=\mu=\Delta=0$ limit, and the remaining definitions,

$$
\begin{aligned}
I_{3}= & \frac{15}{16} \sum_{j= \pm 1} \int \frac{d^{3} k}{(2 \pi)^{3}} \int_{0}^{\infty} \frac{t^{2} d t}{\sqrt{1+t}} \\
& \times \frac{1}{E_{k}^{l}} \frac{j \mu\left(\mathcal{M}-2 j \mu E_{k}^{l}\right)^{3}}{\left[\left(k^{2}+M_{0}^{2}\right) t+\left(E_{k}^{l}+j \mu\right)^{2}+\Delta^{2}\right]^{\frac{7}{2}}},
\end{aligned}
$$

$$
\begin{gathered}
I_{4}=\int \frac{d^{3} k}{(2 \pi)^{3}} \frac{1}{\left(k^{2}+M_{0}^{2}\right)^{\frac{7}{2}}}, \\
I_{5}=\int \frac{d^{3} k}{(2 \pi)^{3}} \frac{k^{2}}{\left(k^{2}+M_{0}^{2}\right)^{\frac{7}{2}}}, \\
I_{6}=\frac{35}{32} \sum_{j= \pm 1} \int \frac{d^{3} k}{(2 \pi)^{3}} \int_{0}^{\infty} \frac{t^{3} d t}{\sqrt{1+t}} \\
\times \frac{j E_{k}^{l}\left(\mathcal{M}-2 j \mu E_{k}^{l}\right)^{4}}{\left[\left(k^{2}+M_{0}^{2}\right) t+\left(E_{k}^{l}+j \mu\right)^{2}+\Delta^{2}\right]^{\frac{9}{2}}}, \\
I_{7}=\frac{3}{4} \int \frac{d^{3} k}{(2 \pi)^{3}} \int_{0}^{\infty} \frac{t d t}{\sqrt{1+t}} \\
\times \frac{\left(M_{0 s}^{2}-M_{s}^{2}\right)^{2}}{\left[\left(k^{2}+M_{0 s}^{2}\right) t+k^{2}+M_{s}^{2}\right]^{\frac{5}{2}}} .
\end{gathered}
$$

Note that integrals $I_{1}$ to $I_{7}$ are all finite and must be performed up to infinity in $k$. This is the fundamental difference between TRS, where we cut the whole integral in the cutoff $\Lambda$, and MSS, where all finite medium contributions are separated and performed for the whole momentum range.

Finally, the MSS expression for the normalized thermodynamic potential reads

$$
\begin{aligned}
\Omega_{\mathrm{NJL}}^{\mathrm{MSS}}\left(\sigma_{l}, \sigma_{s}, \Delta\right)= & \frac{2 \sigma_{l}^{2}+\sigma_{s}^{2}}{8 G}+\frac{K \sigma_{s} \sigma_{l}^{2}}{16 G^{3}}+\left(G+\frac{K \sigma_{s}}{4 G}\right) \frac{\Delta^{2}}{\left(2 G+\frac{K \sigma_{s}}{4 G}\right)^{2}}-2 N_{c}\left\{\tilde{\mathcal{M}} I_{\text {quad }}\left(M_{0}\right)+\frac{M_{s}^{2}-M_{0 s}^{2}}{4} I_{\text {quad }}\left(M_{0 s}\right)\right. \\
& -\frac{1}{4}\left(\tilde{\mathcal{M}}^{2}-4 \mu^{2} \Delta^{2}\right) I_{\log }\left(M_{0}\right)-\frac{M_{s}^{2}-M_{0 s}^{2}}{8} I_{\log }\left(M_{0 s}\right)+\int \frac{d^{3} k}{(2 \pi)^{3}}\left[\frac{\tilde{\mathcal{M}}^{2}-4 \mu^{2} \Delta^{2}}{4\left(E_{k, 0}^{l}\right)^{3}}-\frac{\tilde{\mathcal{M}}}{E_{k, 0}^{l}}-2 E_{k, 0}^{l}\right. \\
& \left.\left.+E_{k}^{s}-E_{k, 0}^{s}-\frac{M_{s}^{2}-M_{0 s}^{2}}{2 E_{k, 0}^{s}}+\frac{\left(M_{s}^{2}-M_{0 s}^{2}\right)^{2}}{8\left(E_{k, 0}^{s}\right)^{3}}+\sum_{j= \pm} \sqrt{\left(E_{k}^{l}+j \mu\right)^{2}+\Delta^{2}}\right]\right\},
\end{aligned}
$$

with the definitions $\tilde{\mathcal{M}}=\Delta^{2}+M_{l}^{2}-M_{0}^{2}, E_{k, 0}=\sqrt{k^{2}+M_{0}^{2}}$, and $E_{k, 0}^{s}=\sqrt{k^{2}+M_{0 s}^{2}}$.

\section{RESULTS AND DISCUSSIONS}

In this paper, we have considered the SU(3) version of the NJL model at finite isospin imbalance incorporating the strange quark sector and the KMT determinant for both the zero and finite temperature cases. Thus, we have extended our previous study of the QCD equation of state at nonzero isospin density and zero temperature within the SU(2) version of the Nambu-Jona-Lasinio model. Besides, in this work, we have also considered for the $\mathrm{SU}(2)$ model the effects of finite temperature in order to perform a complete comparison between the two versions of the NJL model.

The effects of the regularization scheme are discussed in details. We have used two alternative approaches for the regularization of the nonrenormalizable NJL model, the first one, which we have named TRS, is the most frequently found in the literature where the ultraviolet divergences are regularized through a sharp three-dimensional (3D) cutoff, as shown in Sec. II. It is important to point out that the TRS approach does not disentangle finite medium contributions from the infinity vacuum term and physically meaningful contributions are usually discarded. The second scheme, which we named MSS, is capable of disentangling exactly the vacuum divergent term from the finite medium ones and 
only the truly divergent vacuum is regularized through a sharp 3D cutoff. It will be discussed in what follows how dependent are the observables on the chosen regularization scheme and which one is the more appropriated for each particular situation.

The most important first principle approach to QCD in the nonperturbative regime is the lattice QCD simulation. Since for the finite isospin scenario no sign problem is found in the LQCD calculations, whenever possible, our results are compared with recent LQCD data. In order to make possible such comparisons, we have had to fit the $\mathrm{SU}(2)$ and the SU(3) NJL model parameters according to the pion mass and pion decay constant adopted in LQCD calculations. In Tables I and II, our fitted parameters are shown for the SU(2) and SU(3) NJL models, respectively. To compare our results with lattice simulations, we used Set I for zero temperature and Set II for finite temperature cases. The fitting procedure for the $\mathrm{SU}(3)$ version of the NJL model is more involved and due to this fact we have included some details of this parametrization procedure in the Appendix.

Next, we discuss our results for the $\mathrm{SU}(2)$ and $\mathrm{SU}(3)$ NJL models at finite isospin density and zero and finite temperature using the TRS and MSS regularization schemes. At this point, we would like to emphasize the fact that the results for finite isospin and zero temperature within the SU(2) NJL model have been obtained in our previous paper [53]. However, they are shown here for a complete comparison between the NJL model versions as we have extended the zero temperature results for the SU(3) case. The finite temperature extension, however, is a completely new addition in this present work, which has been done here for both $\mathrm{SU}(2)$ and $\mathrm{SU}(3)$ cases.

\section{A. Zero temperature results}

We start by showing our zero temperature SU(3) results, Fig. 1, for the chiral and pion condensates, which have been evaluated, respectively, according to the equations

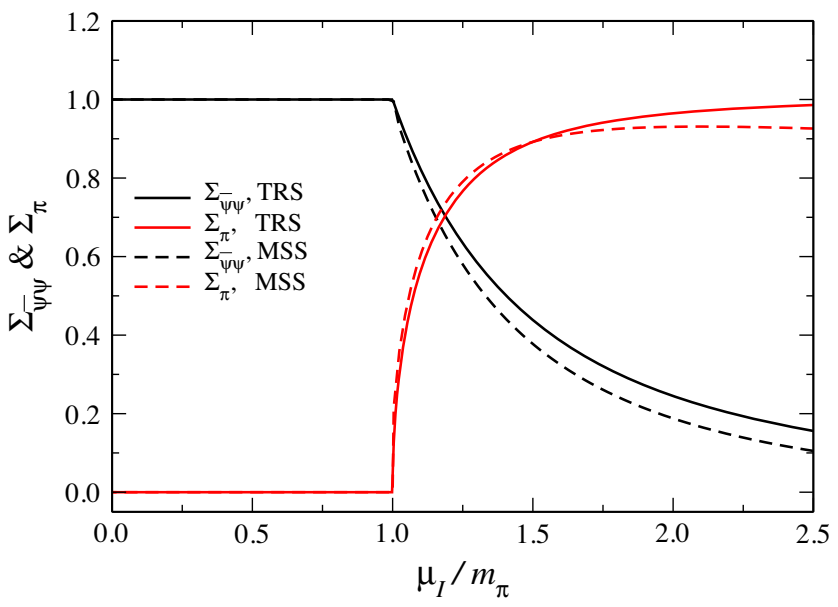

FIG. 1. Chiral and pion condensates as functions of $\mu_{I}$ at $T=0$, evaluated according to Eqs. (3.1) and (3.2), for TRS and MSS.

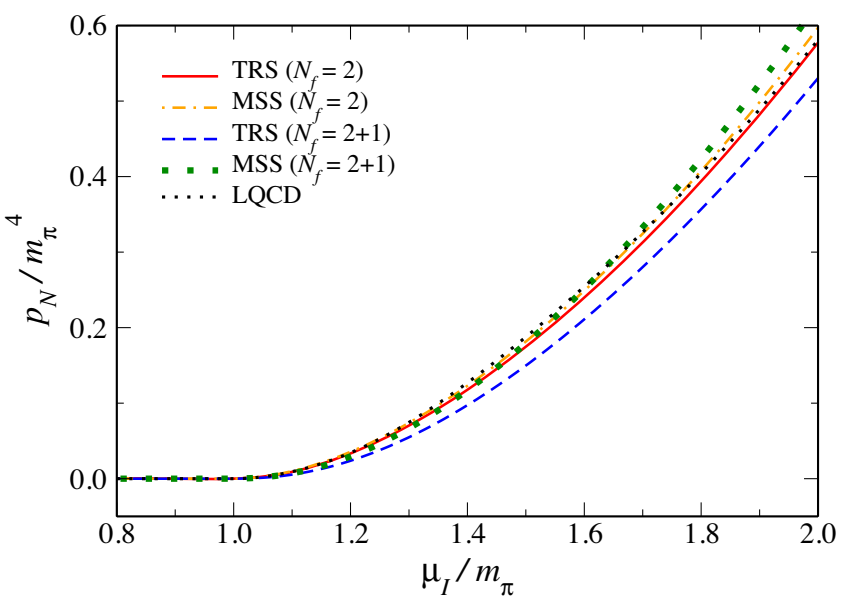

FIG. 2. Normalized pressure $p_{N}$ as a function of $\mu_{I}$ at $T=0$, for SU(2) (solid and dot-dashed lines) and SU(3) (dashed and dotted lines) comparing TRS, MSS, and lattice results from Ref. [54] (small dots).

$$
\begin{gathered}
\Sigma_{\bar{\psi} \psi}=\frac{m_{l}}{m_{\pi}^{2} f_{\pi}^{2}}\left[\frac{\sigma_{l}-\sigma_{l}^{0}}{2 G}\right]+1, \\
\Sigma_{\pi}=\frac{m_{l}}{m_{\pi}^{2} f_{\pi}^{2}} \frac{\Delta}{2 G+K \frac{\sigma_{s}}{4 G}},
\end{gathered}
$$

as a function of the isospin chemical potential $\mu_{I}$ for both the TRS and MSS approaches. Here it is worth to mention that the chiral condensate in Eq. (3.1) does not include the contribution from the strange quarks. We have used the same definitions for these quantities as LQCD [49,52], as we have compared our results against them for the case of finite temperature. ${ }^{3}$ Our results show that the difference between both approaches increases at higher $\mu_{l}$, where the condensates calculated in the MSS scheme are systematically lower than the corresponding ones calculated within the TRS scheme.

The results for the normalized pressure are shown in Fig. 2 as a function of the isospin chemical potential. The normalized pressure $p_{N}$ is defined as

$$
p_{N}=P_{\mathrm{NJL}}\left(T, \mu_{I}\right)-P_{\mathrm{NJL}}\left(T, \mu_{I}=0\right),
$$

where $P_{\mathrm{NJL}}$ is defined in Eq. (2.12). It is apparent of this latter figure that compared to the LQCD results [54], the SU(3)-NJL is better in the MSS scheme (dotted lines) than in the TRS scheme (dashed lines), at least, for $\mu_{I} \leq 2 m_{\pi}$. It is also apparent from Fig. 2 that the $\mathrm{SU}(2)-\mathrm{NJL}$ results [53] agree slightly better with LQCD data than the SU(3) ones

\footnotetext{
${ }^{3}$ Note that there is an apparent $\frac{1}{2}$ factor difference between our definition of the condensates and the definition given in Refs. [49,52], like it has been done in Ref. [20]. This has been done to compensate for the same factor in our definitions of $\sigma_{l}$ and $\Delta$.
} 


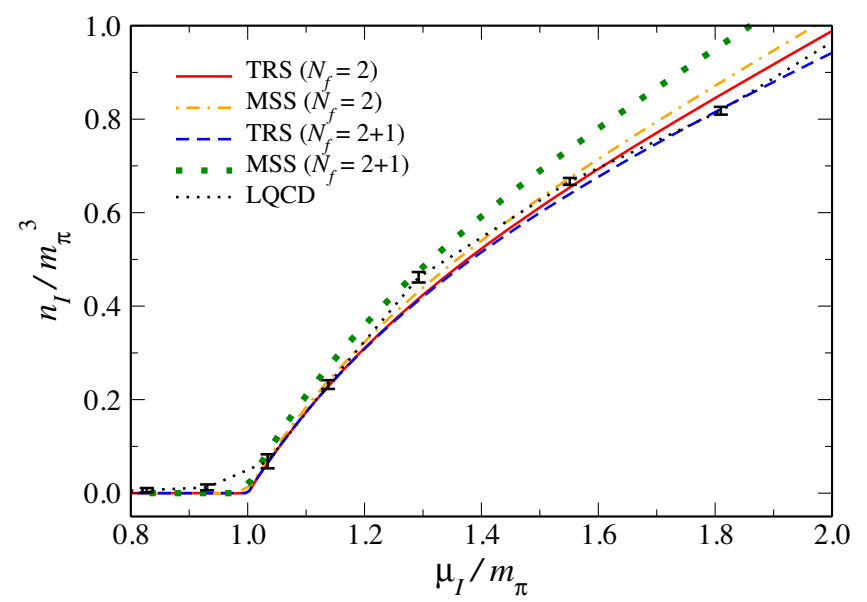

FIG. 3. Isospin density $n_{I}$ as a function of $\mu_{I}$ at $T=0$, for $\mathrm{SU}(2)$ (solid and dot-dashed lines) and SU(3) (dashed and dotted lines) comparing TRS, MSS, and lattice results from Ref. [54] (small dots).

and the differences between TRS and MSS are less important in this case.

The results for the isospin density [see Eq. (2.13)] as a function of $\mu_{I}$ at $T=0$ are shown in Fig. 3. Although the lattice data points are few, one can see that for small $\mu_{I}$ the SU(3)-NJL model using the MSS scheme is in better agreement with LQCD results [54]. Nevertheless, when $\mu_{I}$ increases, the SU(3)-NJL model in the TRS scheme seems to be closer to the LQCD results. As compared to the $\mathrm{SU}(2)-\mathrm{NJL}$ model results, the MSS scheme is closer to lattice data for $\mu_{I} / m_{\pi}<1.6$. Thus, for both $\mathrm{SU}(2)$ and $\mathrm{SU}(3)$ versions of NJL, it seems that for larger isospin chemical potential the TRS scheme is closer to lattice data. If one considers the overall trend of the lattice data, the $\mathrm{SU}(2)-\mathrm{NJL}$ model seems to reproduce better the lattice data, as already noticed in our discussion of the behavior of the pressure. We mention here that it would be highly desirable to get more lattice data in order to better distinguish the differences among the versions of NJL and regularization schemes.

Next up in Fig. 4, we show the scaled energy density [see Eq. (2.13)] as a function of the scaled $\mu_{I}$ chemical potential for the SU(3)-NJL model and $T=0$. The energy density is scaled with its ideal or the Stefan-Boltzmann limit for finite $\mu_{I}$ and $\mu_{B}=T=0$, given by [72]

$$
\varepsilon_{\mathrm{SB}}\left(\mu_{I}\right)=\frac{N_{c} N_{f}}{4 \pi^{2}} \frac{\mu_{I}^{4}}{16}
$$

A comparison with the perturbative QCD calculation [72] is performed for larger values of $\mu_{I}$. Some care is necessary when using NJL for larger $\mu_{I}$, since we have the $\Lambda$ cutoff as a natural momentum scale which makes the model quantitatively trusty for scales $\leq 4 m_{\pi}$; nevertheless, only qualitative conclusions can be done extrapolating such limit.

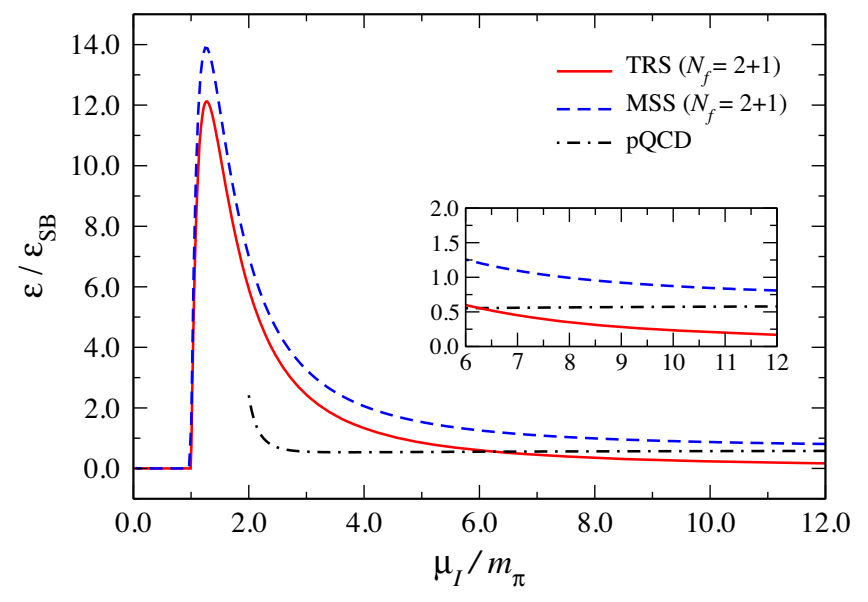

FIG. 4. Comparison of the energy density scaled by the StefanBoltzmann limit for zero temperature and finite isospin chemical potential. The pQCD results for higher values of $\mu_{I} / m_{\pi}$ are obtained using the expressions from Ref. [72].

However, it is clear from the latter figure that the MSS scheme follows the trend of $\mathrm{pQCD}$ for higher values of $\mu_{I}$. Moreover, one clearly sees in the inset of Fig. 4 that the Stefan-Boltzmann limit is expected to be achieved only in the MSS scheme.

We finish our discussions on the zero temperature results showing in Fig. 5 the normalized interaction measure $\Delta I$ as a function of the scaled isospin chemical potential. The normalized interaction measure $\Delta I$ is defined as

$$
\Delta I=I_{\mathrm{NJL}}\left(T, \mu_{I}\right)-I_{\mathrm{NJL}}\left(T, \mu_{I}=0\right),
$$

where $I_{\mathrm{NJL}}$ is defined in Eq. (2.15). As can be seen from Fig. 5, the difference between the TRS (solid and dashed lines) and MSS (dot-dashed and dotted lines) results for

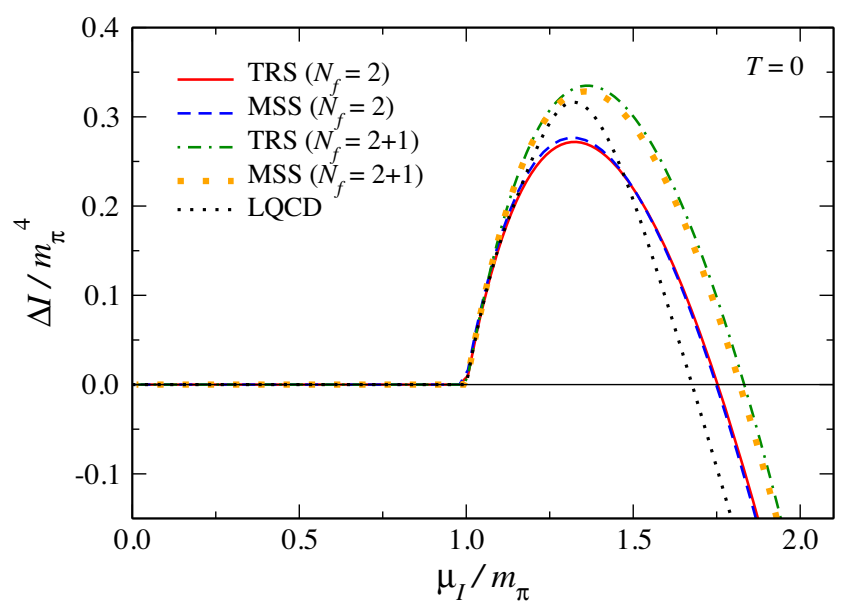

FIG. 5. Normalized interaction measure $(\Delta I)$ as a function of $\mu_{I}$ for SU(2) (solid and dot-dashed lines) and SU(3) (dashed and dotted lines) comparing TRS, MSS, and lattice results from Refs. [54,66] (small dots). 
both $\mathrm{SU}(2)$ and $\mathrm{SU}(3)$ is small, but the MSS results appear closer to the LQCD results $[54,66]$.

At zero temperature, we observe the general trend that the agreement between lattice and NJL results is better for two flavor at larger values of $\mu_{I}$ and for three flavor at lower values of $\mu_{I}$. Similar observations can also be found in recent $\chi \mathrm{PT}$ studies $[19,20]$. In the next subsection, we discuss the results at finite temperature and isospin chemical potential.

\section{B. Finite temperature results}

At finite temperature, we begin with Fig. 6, where the chiral and pion condensates as a function of the temperature calculated at fixed isospin chemical potential $\left(\mu_{I}=0.206 \mathrm{GeV}\right)$ are shown. In the latter figure, results for the SU(3)-NJL model using both the MSS and TRS schemes are compared. One clearly sees that the melting temperature is larger in the MSS scheme compared to the TRS one for both condensates. However, the pion condensate melting is a second order phase transition with the critical temperature $\sim 158 \mathrm{MeV}$ for TRS and $\sim 173 \mathrm{MeV}$ for MSS. On the other hand, the corresponding chiral condensate behavior signals a crossover. Below a certain temperature (around $\sim 165 \mathrm{MeV}$ for $\mu_{I}=206 \mathrm{MeV}$ ) the TRS result for the chiral condensate supersedes the MSS result, whereas after that temperature the TRS result decreases more rapidly than the MSS result.

Next, we discuss about our findings for the thermodynamic quantities. In Fig. 7, the results are shown for the normalized pressure, Eq. (3.3), as a function of the isospin chemical potential for three different temperatures, which have been chosen because they are available in LQCD simulations $[49,52,64,65]$. The results have been shown for both SU(2) and SU(3)-NJL model within the TRS and MSS schemes. For larger values of $\mu_{I}$, the SU(3)-NJL model within the TRS scheme is closer to the LQCD results, although the agreement is better for lower temperatures.

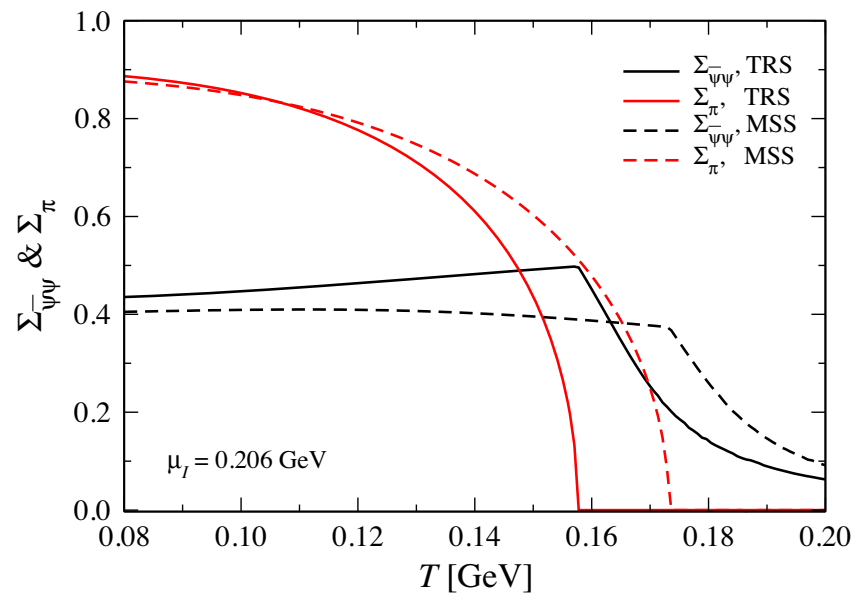

FIG. 6. SU(3) chiral and pion condensates as functions of the temperature $T$ with $\mu_{I}=0.206 \mathrm{GeV}$ for TRS and MSS.
For each of the plots, we have also displayed an inset, where we show the results for smaller values of $\mu_{I}$ $\left(0.4 m_{\pi}<\mu_{I}<1.6 m_{\pi}\right)$ and in this case we observe that the MSS and TRS schemes are closer to each other, and for the largest temperature considered in this work the SU(3)-NJL using the MSS scheme is slightly better. Here, we notice a different behavior for finite temperature when compared to the zero temperature case. For $T=0$, the
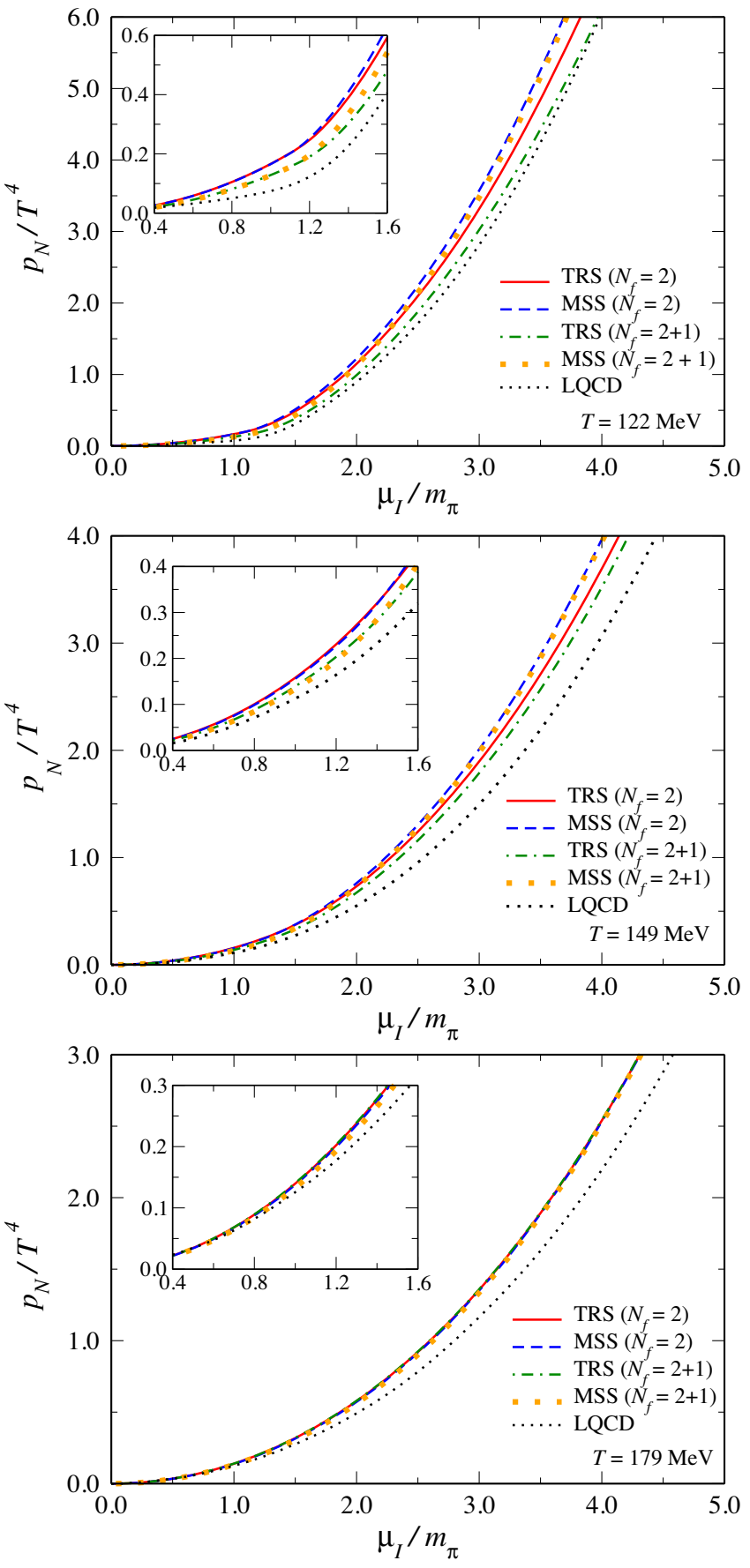

FIG. 7. Normalized pressure $p_{N}$ as a function of $\mu_{I}$ for different temperatures $T$, for TRS and MSS, comparing SU(2) and SU(3) to lattice results from Refs. $[49,52,64,65]$. 
$\mathrm{SU}(2)-\mathrm{NJL}$ results for the $p_{N}$ are in general in better agreement with LQCD results (for TRS and MSS) than the SU(3) NJL model.

In Fig. 8, the isospin density as a function of isospin chemical potential is plotted for the same three temperatures as discussed above. We can observe a change of slope in the isospin density for $T=122 \mathrm{MeV}$ and $T=$ $149 \mathrm{MeV}$ which is related to the formation of the pion
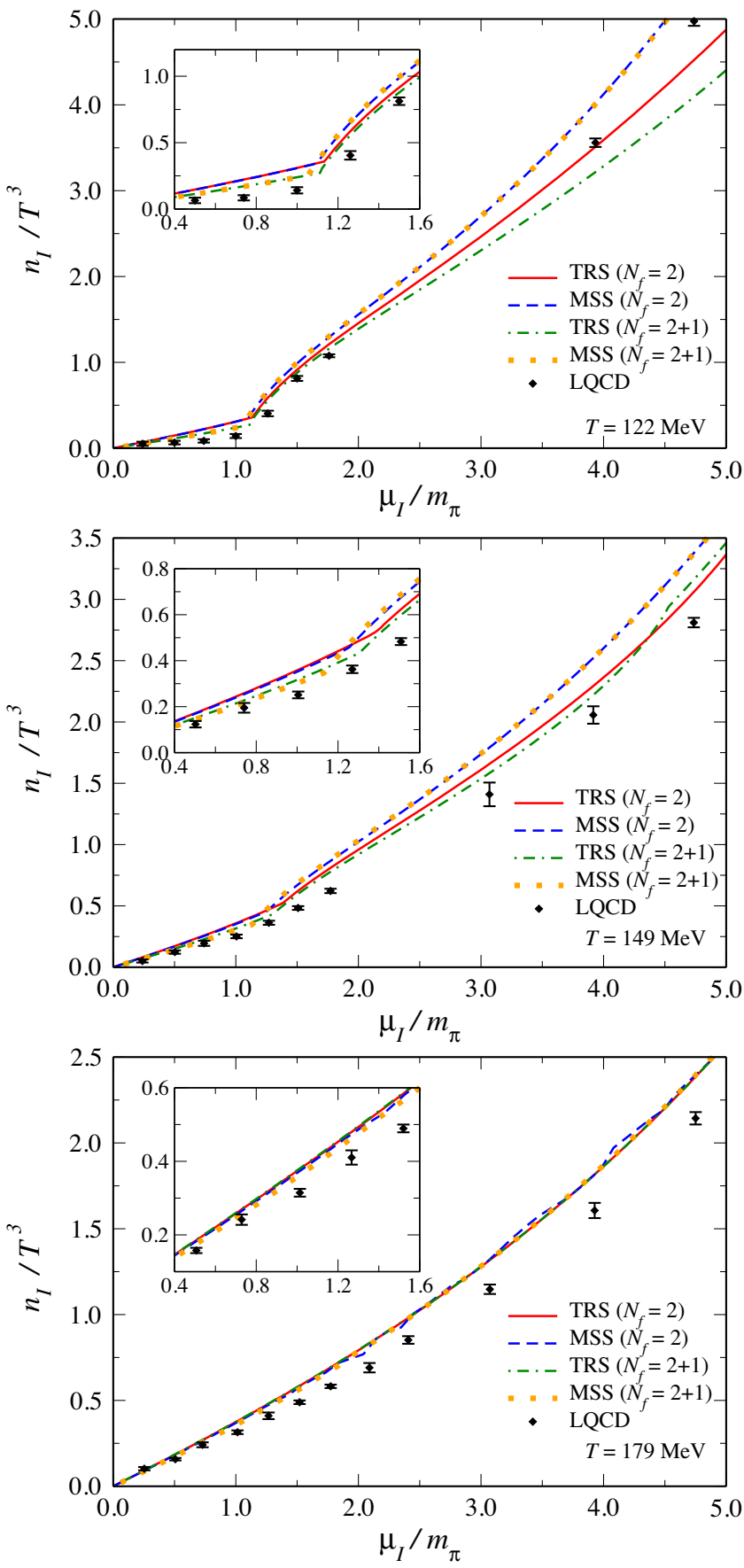

FIG. 8. Isospin density $n_{I}$ as a function of $\mu_{I}$ for different temperatures $T$, for TRS and MSS, comparing SU(2) and SU(3) to lattice results from Refs. [49,52,64,65]. condensate, when $\Delta$ becomes nonzero. This change is not present in the curves correspondent to $T=179 \mathrm{MeV}$ because this temperature is high enough to prevent the pion condensate to be formed. In this case, we have a similar behavior as obtained for the normalized pressure, i.e., the SU(3)-NJL with the TRS scheme gives a better overall agreement with the LQCD results $[49,52,64,65]$.
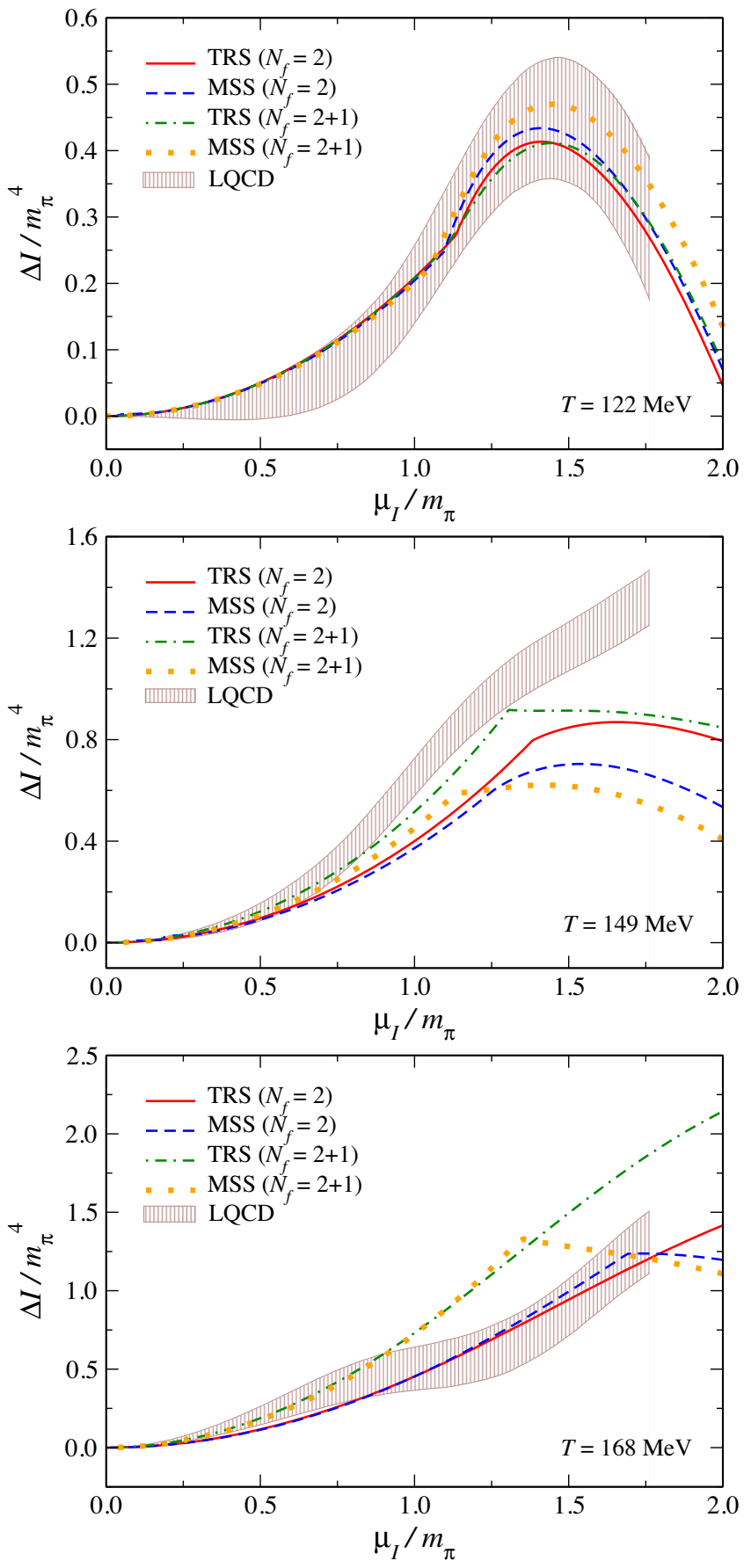

FIG. 9. Normalized trace anomaly or interaction measure $(\Delta I)$ as a function of $\mu_{I}$ for different temperatures $T$, for TRS and MSS, comparing $\mathrm{SU}(2)$ and $\mathrm{SU}(3)$ to lattice results from Ref. [66]. 

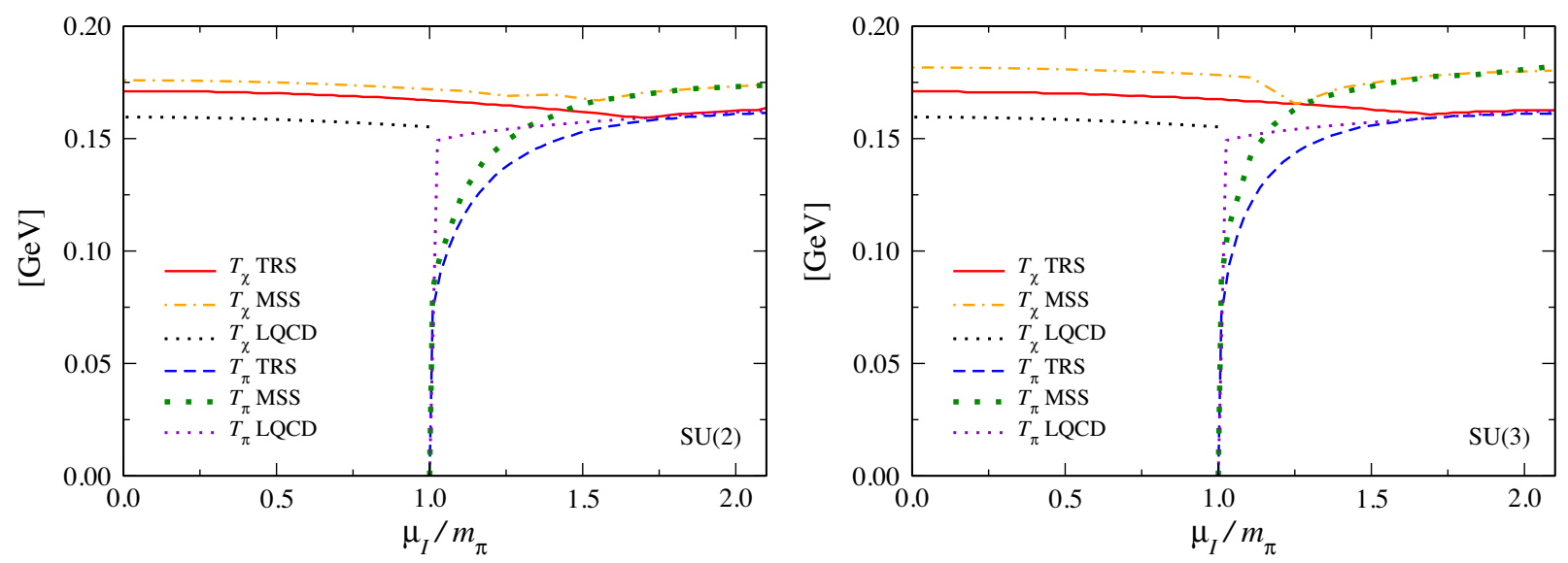

FIG. 10. $T-\mu_{I}$ phase diagram within NJL model, implementing both TRS and MSS and comparing SU(2) and SU(3) to lattice results from Refs. [49,52,64,65].

For the lower temperature $(T=122 \mathrm{MeV})$ considered here, the SU(2)-NJL in TRS scheme gives good results. As before, the inset plots show that the MSS and TRS are very similar for low isospin chemical potential and the SU(3)-NJL is the better model. At this point, we once again stress the fact that the LQCD data for the isospin density are not conclusive enough because of its scarcity and oscillating nature.

Within the thermodynamic quantities we have also shown the variation of the normalized interaction measure $\Delta I$ [see Eq. (3.5)] as a function of isospin chemical potential for three different temperatures in Fig. 9. In this case though, instead of $T=179 \mathrm{MeV}$, the highest temperature we choose is $T=168 \mathrm{MeV}$, based on the availability of LQCD results [66]. For lower temperature $(T=122 \mathrm{MeV})$, it is apparent from Fig. 9 that both TRS and MSS results for $\mathrm{SU}(2)$ and $\mathrm{SU}(3)$ fall within the domain prescribed by LQCD. At $T=149 \mathrm{MeV}$, for very low $\mu_{I}$, all the NJL results fall within the LQCD domain; however, with increasing $\mu_{I}$, only SU(3) TRS result appears closer to LQCD result. For the higher temperature considered in Fig. $9(T=168 \mathrm{MeV})$, we can see that the SU(2)-NJL results for TRS and MSS are more in agreement with the LQCD results. It is worth to mention here that for $T=168 \mathrm{MeV}$ at higher values of $\mu_{I}$ the pion condensate is zero in TRS, which is not the case of MSS. The presence of a nonzero $\Delta$ makes both pressure and energy density smaller, and the change of slope is related to the isospin density, as we discussed before (see Fig. 8).

We conclude our discussion for finite temperature with the $T-\mu_{I}$ phase diagrams shown in Fig. 10 for both $\mathrm{SU}(2)$ and SU(3). In each of the phase diagrams, we have considered TRS and MSS results within NJL and compared them with the available LQCD results $[49,52,64,65]$. We notice that for both SU(2) and SU(3), TRS results appear closer to LQCD for pion condensation and chiral crossover, compared to MSS. For $\mu_{I}=0$, the critical temperature for the chiral phase transition within NJL is higher than the same within LQCD, with maximum quantitative difference of $\sim 16 \mathrm{MeV}$ for $\mathrm{SU}(2)$ and $\sim 22 \mathrm{MeV}$ for SU(3). On the other hand, investigating the pseudo triple point, beyond which both the phase transitions coincide, we find that the pseudo triple points within MSS are closer to the LQCD results compared to TRS, specially for SU(3)-NJL.

\section{ACKNOWLEDGMENTS}

We thank Gergely Endrodi and Bastian B. Brandt for useful discussions and also for providing the necessary lattice data sets, both at zero and finite temperatures. We also thank Pedro Costa for useful discussions regarding the SU(3) parametrization. This work was partially supported by Conselho Nacional de Desenvolvimento Científico e Tecnológico (CNPq) under Grants No. 304758/2017-5 (R. L.S.F), No. 304518/2019-0 (S. S. A), and No. 149831/2020-0 (B.S. L.); as a part of the project INCT-FNA (Instituto Nacional de Ciência e Tecnologia-Física Nuclear e Aplicações) No. 464898/ 2014-5 (S. S. A); U.S. DOE under Grant No. DE-FG0200ER41132 and Fundação de Amparo à Pesquisa do Estado de São Paulo under Grant No. 2017/26111-4 (D.C.D); and Fundação de Amparo à Pesquisa do Estado do Rio Grande do Sul (FAPERGS), Grants No. 19/2551-0000690-0 and No. 19/2551-0001948-3 (R. L. S. F.). D.C. D. acknowledges the support of the Simons Foundation under the Multifarious Minds Program Grant No. 557037. A. B. acknowledges the support from Guangdong Major Project of Basic and Applied Basic Research No. 2020B0301030008 and Science and Technology Program of Guangzhou Project No. 2019050001.

\section{APPENDIX: NJL SU(3) PARAMETRIZATION}

In this appendix, the procedure used for the parametrization of the NJL SU(3) model is discussed. Although we use standard techniques, the expressions are not usually 
given in a friendly way in the literature [73-75]. Moreover, having in mind to allow the reader to reproduce our calculations, the essential expressions are given here. In order to restrict the number of free parameters, we set the $\mathrm{u}$ and d current quark masses equal, i.e., $m_{u}=m_{d}$. Therefore, we obtain by solving the self-consistent gap equations that the corresponding constituent $\mathrm{u}$ and $\mathrm{d}$ quark masses are also equal to each other, $M_{u}=M_{d}$. The free model parameters are the coupling constants $G$ and $K$, the current s quark mass $m_{s}$, and the cutoff parameter $\Lambda$. The value of the $m_{u}=m_{d}$ current quark mass is arbitrarily fixed. The observables that are used in the fitting procedure are the pion mass, the pion decay constant, the kaon mass, and the $\eta^{\prime}$ meson mass.

\section{Gap equations}

The gap equations for the NJL SU(3) model are given by

$$
M_{i}=m_{i}-4 G \sigma_{i}+2 K \sigma_{j} \sigma_{k},
$$

with $(i j k)$ being any cyclic permutation of $(u, d, s)$, and the condensate is defined as

$$
\sigma_{i}=\left\langle\bar{q}_{i} q_{i}\right\rangle=-i \operatorname{Tr}\left[S_{i}(p)\right]=-i \operatorname{Tr} \frac{1}{\not p-M_{i}} .
$$

After the explicit calculation of the trace in Dirac and color spaces, one obtains

$$
\sigma_{i}=\left\langle\bar{q}_{i} q_{i}\right\rangle=-4 M_{i} I_{1}^{i},
$$

with

$I_{1}^{i}=\frac{N_{c}}{4 \pi^{2}} \int_{0}^{\Lambda} \frac{p^{2} d p}{E_{i}}=\frac{N_{c}}{8 \pi^{2}}\left[\Lambda \epsilon_{i}^{\Lambda}-M_{i}^{2} \ln \left(\frac{\Lambda+\epsilon_{i}^{\Lambda}}{M_{i}}\right)\right]$,

where $E_{i}=\sqrt{p^{2}+M_{i}^{2}}$ and $\epsilon_{i}^{\Lambda}=\sqrt{\Lambda^{2}+M_{i}^{2}}, i=(u, d, s)$.

\section{Pion and kaon masses and decay constants}

The dispersion relations for the pion and kaon masses are given by

$$
\begin{aligned}
& 1-\left.2 G_{\pi} \Pi_{\pi}^{p}\left(Q^{2}\right)\right|_{Q^{2}=m_{\pi}^{2}}=0, \\
& 1-\left.2 G_{K} \Pi_{K}^{p}\left(Q^{2}\right)\right|_{Q^{2}=m_{K}^{2}}=0,
\end{aligned}
$$

where $\Pi_{\pi}^{p}=\Pi_{u u}^{p}+\Pi_{d d}^{p}=2 \Pi_{u u}^{p}$ and $\Pi_{K}^{p}=2 \Pi_{u s}^{p}$ are the pseudoscalar polarization loops for the pion and kaon mesons, respectively, which can be evaluated from the general expression

$$
\Pi_{i j}^{p}\left(Q^{2}\right)=2\left(\left(I_{1}^{i}+I_{1}^{j}\right)-\left[Q^{2}-\left(M_{i}-M_{j}\right)^{2}\right] I_{2}^{i j}\right),
$$

with $I_{1}^{i}$ already given in Eq. (A3) and $I_{2}^{i j}$ is given by

$$
I_{2}^{i j}\left(Q^{2}\right)=\frac{N_{c}}{4 \pi^{2}} \int_{0}^{\Lambda} \frac{p^{2} d p}{E_{i} E_{j}} \frac{E_{i}+E_{j}}{Q^{2}-\left(E_{i}+E_{j}\right)^{2}},
$$

whereas the modified couplings $G_{\pi}$ and $G_{K}$ for pions and kaons, respectively, are given by

$$
\begin{aligned}
G_{\pi} & =G-\frac{1}{2} K \sigma_{s}, \\
G_{K} & =G-\frac{1}{2} K \sigma_{u} .
\end{aligned}
$$

For the pion decay constant, we have

$$
\begin{aligned}
f_{\pi}= & g_{\pi \bar{q} q} \frac{Q_{\mu}}{Q^{2}} i N_{c} \int d^{4} p \operatorname{Tr}\left[\gamma^{\mu} \gamma^{5} S_{u}\left(p+\frac{Q}{2}\right)\right. \\
& \left.\times \gamma^{5} S_{u}\left(p+\frac{Q}{2}\right)\right],
\end{aligned}
$$

and after the explicit calculation of the trace in the last equation, one obtains

$$
f_{\pi}=-4 M_{u} g_{\pi \bar{q} q}\left(m_{\pi}^{2}\right) I_{2}^{u u}\left(m_{\pi}^{2}\right),
$$

where the coupling strength for the meson-quark-quark interaction is given by

$$
g_{\pi \bar{q} q}^{-2}\left(m_{\pi}^{2}\right)=\left.\frac{\partial \Pi_{\pi}^{p}}{\partial Q^{2}}\right|_{Q^{2}=m_{\pi}^{2}}=\left.2 \frac{\partial \Pi_{u u}^{p}}{\partial Q^{2}}\right|_{Q^{2}=m_{\pi}^{2}} .
$$

The last expression follows from the derivative of Eq. (A6),

$$
\frac{\partial \Pi_{u u}^{p}}{\partial Q^{2}}=-2 I_{2}^{u u}-2 Q^{2} \frac{\partial}{\partial Q^{2}} I_{2}^{u u} .
$$

\section{3. $\boldsymbol{\eta}$ and $\boldsymbol{\eta}^{\prime}$ mesons}

For the case of $\eta$ and $\eta^{\prime}$ meson, the inverse mesonic propagator assumes a matrix form [73]

$$
D^{-1}=\frac{1}{2} K^{-1}-\Pi,
$$

where the effective coupling matrix $K$ and the mesonic selfenergy $\Pi$ for the $\eta-\eta^{\prime}$ system are given by the matrices

$$
\begin{aligned}
\Pi & =\left(\begin{array}{ll}
\Pi_{00} & \Pi_{08} \\
\Pi_{08} & \Pi_{88}
\end{array}\right), \quad K=\left(\begin{array}{ll}
K_{00} & K_{08} \\
K_{08} & K_{88}
\end{array}\right), \\
K^{-1} & =\frac{1}{\operatorname{det} K}\left(\begin{array}{cc}
K_{88} & -K_{08} \\
-K_{08} & K_{00}
\end{array}\right),
\end{aligned}
$$

where the determinant of $K$ is given by det $K=K_{00} K_{88}-$ $K_{08}^{2}$ and the specific components are given by 


$$
\begin{gathered}
K_{00}=G+\frac{1}{3} K\left(2 \sigma_{u}+\sigma_{s}\right), \\
K_{88}=G-\frac{1}{6} K\left(4 \sigma_{u}-\sigma_{s}\right), \\
K_{08}=K_{80}=-\frac{\sqrt{2}}{6} K\left(\sigma_{u}-\sigma_{s}\right),
\end{gathered}
$$

and

$$
\begin{gathered}
\Pi_{00}=\frac{2}{3}\left[2 \Pi_{u u}^{p}(Q)+\Pi_{s s}^{p}(Q)\right], \\
\Pi_{88}=\frac{2}{3}\left[\Pi_{u u}^{p}(Q)+2 \Pi_{s s}^{p}(Q)\right], \\
\Pi_{08}=\Pi_{80}=\frac{2 \sqrt{2}}{3}\left[\Pi_{u u}^{p}(Q)-\Pi_{s s}^{p}(Q)\right] .
\end{gathered}
$$

So, finally the inverse propagator is given by

$$
D^{-1}=\frac{1}{2 \operatorname{det} K}\left(\begin{array}{cc}
A & B \\
B & C
\end{array}\right)
$$

with

$$
\begin{gathered}
A=K_{88}-2 \operatorname{det} K \Pi_{00}, \\
B=-\left(K_{08}+2 \operatorname{det} K \Pi_{08}\right), \\
C=K_{00}-2 \operatorname{det} K \Pi_{88} .
\end{gathered}
$$

Diagonalizing $D^{-1}$, we get

$$
D^{-1}=\frac{1}{2 \operatorname{det} K} \mathcal{O}^{-1}\left(\begin{array}{cc}
D_{\eta}^{-1} & 0 \\
0 & D_{\eta^{\prime}}^{-1}
\end{array}\right) \mathcal{O}
$$

where the diagonalizing orthogonal matrix $\mathcal{O}$ is given by

$$
\mathcal{O}=\left(\begin{array}{cc}
\cos \theta_{p} & \sin \theta_{p} \\
-\sin \theta_{p} & \cos \theta_{p}
\end{array}\right), \quad \tan \left(2 \theta_{p}\right)=\frac{2 B}{A-C}
$$

and the diagonal elements now represent the dispersion relations for $\eta$ and $\eta^{\prime}$ mesons, i.e.,

$$
\begin{aligned}
& D_{\eta}^{-1}=(A+C)-\sqrt{(C-A)^{2}+4 B^{2}}, \\
& D_{\eta^{\prime}}^{-1}=(A+C)+\sqrt{(C-A)^{2}+4 B^{2}} .
\end{aligned}
$$

The masses of the $\eta$ and $\eta^{\prime}$ meson can now be determined from the equations

$$
\begin{aligned}
& D_{\eta}^{-1}\left(Q=m_{\eta}\right)=0, \\
& D_{\eta^{\prime}}^{-1}\left(Q=m_{\eta^{\prime}}\right)=0 .
\end{aligned}
$$

At this point, we want to note that $m_{\eta}$ can be evaluated from Eq. (A27) by using the integrals from Eqs. (A3) and (A7). But as $\eta^{\prime}$, in general, exists above the $\bar{q} q$ continuum, Eq. (A28) has complex poles, which we can assume to be of the form, $Q=Q_{0}=m_{\eta^{\prime}}-\frac{1}{2} i \Gamma$, with $\Gamma$ being the width of the $\eta^{\prime}$ resonance. In the latter case, the calculation of $A, B$, and $C$ in Eq. (A26) can be readily done making in Eq. (A6) the replacement

$$
Q^{2} I_{2}^{i i} \rightarrow\left[m_{\eta^{\prime}}^{2} \operatorname{Re} I_{2}^{i i}+m_{\eta^{\prime}} \Gamma \operatorname{Im} I_{2}^{i i}\right]+i\left[m_{\eta^{\prime}}^{2} \operatorname{Im} I_{2}^{i i}-m_{\eta^{\prime}} \Gamma \operatorname{Re} I_{2}^{i i}\right]
$$

where terms of order $\Gamma^{2}$ have been neglected [74]. (For some other studies where $\Gamma^{2}$ terms have not been neglected, see [75].) Then, after substituting the latter expression in Eq. (A6), one obtains

$$
\Pi_{i i}^{p}\left(Q^{2}\right)=\operatorname{Re} \Pi_{i i}^{p}\left(Q^{2}\right)+i \operatorname{Im} \Pi_{i i}^{p}\left(Q^{2}\right)
$$

where

$\operatorname{Re} \Pi_{i i}^{p}\left(Q^{2}\right)=4 I_{1}^{i}-2\left(m_{\eta^{\prime}}^{2} \operatorname{Re} I_{2}^{i i}+m_{\eta^{\prime}} \Gamma \operatorname{Im} I_{2}^{i i}\right)$,

$\operatorname{Im} \Pi_{i i}^{p}\left(Q^{2}\right)=2\left(m_{\eta^{\prime}} \Gamma \operatorname{Re} I_{2}^{i i}-m_{\eta^{\prime}}^{2} \operatorname{Im} I_{2}^{i i}\right)$.

The real and imaginary parts of the integral $I_{2}^{i i}$ can be obtained from Eq. (A7) by using the Sokhotski-Plemelj formula

$$
\lim _{\epsilon \rightarrow 0} \frac{1}{x-i \epsilon}=\mathcal{P} \frac{1}{x}+i \pi \delta(x)
$$

where $\mathcal{P}$ stands for the Cauchy principal value and this formula makes sense only when integrated in $x$. Then, one obtains

$$
\begin{aligned}
& \operatorname{Re} I_{2}^{i i}(Q)=\frac{N_{c}}{2 \pi^{2}} \mathcal{P} \int_{0}^{\Lambda} \frac{p^{2} d p}{E_{i}} \frac{1}{Q^{2}-4 E_{i}^{2}} \\
& \operatorname{Im} I_{2}^{i i}(Q)=\frac{N_{c}}{16 \pi} \sqrt{1-\frac{4 M_{i}^{2}}{Q^{2}}},
\end{aligned}
$$

where in these expressions we assume that $Q=m_{\eta^{\prime}}$ and $m_{\eta^{\prime}}>2 M_{i}$. 
[1] K. Fukushima and T. Hatsuda, Rep. Prog. Phys. 74, 014001 (2011).

[2] M. G. Alford, A. Schmitt, K. Rajagopal, and T. Schfer, Rev. Mod. Phys. 80, 1455 (2008).

[3] F. Karsch, Lect. Notes Phys. 583, 209 (2002).

[4] S. Muroya, A. Nakamura, C. Nonaka, and T. Takaishi, Prog. Theor. Phys. 110, 615 (2003).

[5] P. F. Bedaque, EPJ Web Conf. 175, 01020 (2018).

[6] J. B. Kogut and D. K. Sinclair, Phys. Rev. D 66, 034505 (2002).

[7] J. B. Kogut and D. K. Sinclair, Phys. Rev. D 66, 014508 (2002).

[8] D. T. Son and M. A. Stephanov, Phys. Rev. Lett. 86, 592 (2001).

[9] D. T. Son and M. A. Stephanov, Yad. Fiz. 64, 899 (2001) [Phys. At. Nucl. 64, 834 (2001)].

[10] L. Lepori and M. Mannarelli, Phys. Rev. D 99, 096011 (2019).

[11] S. Carignano, L. Lepori, A. Mammarella, M. Mannarelli, and G. Pagliaroli, Eur. Phys. J. A 53, 35 (2017).

[12] O. Janssen, M. Kieburg, K. Splittorff, J. J. M. Verbaarschot, and S. Zafeiropoulos, Phys. Rev. D 93, 094502 (2016).

[13] T. D. Cohen and S. Sen, Nucl. Phys. A942, 39 (2015).

[14] E. S. Fraga, L. F. Palhares, and C. Villavicencio, Phys. Rev. D 79, 014021 (2009).

[15] M. Loewe and C. Villavicencio, Phys. Rev. D 67, 074034 (2003).

[16] M. Loewe and C. Villavicencio, Phys. Rev. D 71, 094001 (2005).

[17] K. Splittorff, D. T. Son, and M. A. Stephanov, Phys. Rev. D 64, 016003 (2001).

[18] P. Adhikari, J. O. Andersen, and P. Kneschke, Eur. Phys. J. C 79, 874 (2019).

[19] P. Adhikari and J. O. Andersen, J. High Energy Phys. 06 (2020) 170.

[20] P. Adhikari and J. O. Andersen, Eur. Phys. J. C 80, 1028 (2020).

[21] J. O. Andersen, N. Haque, M. G. Mustafa, and M. Strickland, Phys. Rev. D 93, 054045 (2016).

[22] D. Ebert, T. G. Khunjua, and K. G. Klimenko, Phys. Rev. D 94, 116016 (2016).

[23] T. G. Khunjua, K. G. Klimenko, R. N. Zhokhov, and V. C. Zhukovsky, Phys. Rev. D 95, 105010 (2017).

[24] T. G. Khunjua, K. G. Klimenko, and R. N. Zhokhov, Phys. Rev. D 98, 054030 (2018).

[25] T. G. Khunjua, K. G. Klimenko, and R. N. Zhokhov, Eur. Phys. J. C 79, 151 (2019).

[26] T. G. Khunjua, K. G. Klimenko, and R. N. Zhokhov, J. High Energy Phys. 06 (2019) 006.

[27] T. G. Khunjua, K. G. Klimenko, and R. N. Zhokhov, Phys. Rev. D 100, 034009 (2019).

[28] T. G. Khunjua, K. G. Klimenko, and R. N. Zhokhov, J. High Energy Phys. 06 (2020) 148.

[29] T. Xia, L. He, and P. Zhuang, Phys. Rev. D 88, 056013 (2013).

[30] C. F. Mu, L. He, and Y. X. Liu, Phys. Rev. D 82, 056006 (2010).

[31] H. Abuki, R. Anglani, R. Gatto, M. Pellicoro, and M. Ruggieri, Phys. Rev. D 79, 034032 (2009).
[32] J. O. Andersen and L. Kyllingstad, J. Phys. G 37, 015003 (2010).

[33] G. f. Sun, L. He, and P. Zhuang, Phys. Rev. D 75, 096004 (2007).

[34] D. Ebert and K. G. Klimenko, Eur. Phys. J. C 46, 771 (2006).

[35] D. Ebert and K. G. Klimenko, J. Phys. G 32, 599 (2006).

[36] L. He, M. Jin, and P. Zhuang, Phys. Rev. D 74, 036005 (2006).

[37] L. He, M. Jin, and P. f. Zhuang, Phys. Rev. D 71, 116001 (2005).

[38] L. He and P. Zhuang, Phys. Lett. B 615, 93 (2005).

[39] A. Barducci, R. Casalbuoni, G. Pettini, and L. Ravagli, Phys. Rev. D 69, 096004 (2004).

[40] D. Toublan and J. B. Kogut, Phys. Lett. B 564, 212 (2003).

[41] M. Frank, M. Buballa, and M. Oertel, Phys. Lett. B 562, 221 (2003).

[42] Z. Y. Lu, C. J. Xia, and M. Ruggieri, Eur. Phys. J. C 80, 46 (2020).

[43] S. Mukherjee, M. G. Mustafa, and R. Ray, Phys. Rev. D 75, 094015 (2007).

[44] A. Bhattacharyya, S. K. Ghosh, A. Lahiri, S. Majumder, S. Raha, and R. Ray, Phys. Rev. C 89, 064905 (2014).

[45] P. Adhikari, J. O. Andersen, and P. Kneschke, Phys. Rev. D 98, 074016 (2018).

[46] R. Stiele, E. S. Fraga, and J. Schaffner-Bielich, Phys. Lett. B 729, 72 (2014).

[47] H. Ueda, T. Z. Nakano, A. Ohnishi, M. Ruggieri, and K. Sumiyoshi, Phys. Rev. D 88, 074006 (2013).

[48] K. Kamikado, N. Strodthoff, L. von Smekal, and J. Wambach, Phys. Lett. B 718, 1044 (2013).

[49] B. B. Brandt, G. Endrödi, and S. Schmalzbauer, Phys. Rev. D 97, 054514 (2018).

[50] B. B. Brandt, G. Endrödi, and S. Schmalzbauer, EPJ Web Conf. 175, 07020 (2018).

[51] B. B. Brandt and G. Endrödi, Proc. Sci., LATTICE2016 (2016) 039.

[52] B. B. Brandt, G. Endrodi, and S. Schmalzbauer, Proc. Sci., Confinement2018 (2018) 260.

[53] S. S. Avancini, A. Bandyopadhyay, D. C. Duarte, and R. L. Farias, Phys. Rev. D 100, 116002 (2019).

[54] B. B. Brandt, G. Endrödi, E. S. Fraga, M. Hippert, J. Schaffner-Bielich, and S. Schmalzbauer, Phys. Rev. D 98, 094510 (2018).

[55] J. A. Wheeler, Phys. Rev. 97, 511 (1955).

[56] D. J. Kaup, Phys. Rev. 172, 1331 (1968).

[57] P. Jetzer, Phys. Rep. 220, 163 (1992).

[58] M. Colpi, S. L. Shapiro, and I. Wasserman, Phys. Rev. Lett. 57, 2485 (1986).

[59] S. L. Liebling and C. Palenzuela, Living Rev. Relativity 15, 6 (2012); 20, 5 (2017).

[60] J. O. Andersen and P. Kneschke, arXiv:1807.08951.

[61] H. Abuki, T. Brauner, and H. J. Warringa, Eur. Phys. J. C 64, 123 (2009).

[62] D. J. Schwarz and M. Stuke, J. Cosmol. Astropart. Phys. 11 (2009) 025; 10 (2010) E01.

[63] M. M. Wygas, I. M. Oldengott, D. Bdeker, and D. J. Schwarz, Phys. Rev. Lett. 121, 201302 (2018). 
[64] B. B. Brandt and G. Endrödi, Phys. Rev. D 99, 014518 (2019).

[65] B. B. Brandt and G. Endrödi (private communication).

[66] V. Vovchenko, B. B. Brandt, F. Cuteri, G. Endrődi, F. Hajkarim, and J. Schaffner-Bielich, Phys. Rev. Lett. 126, 012701 (2021).

[67] R. L. S. Farias, G. Dallabona, G. Krein, and O. A. Battistel, Phys. Rev. C 73, 018201 (2006).

[68] R. L. S. Farias, G. Dallabona, G. Krein, and O. A. Battistel, Phys. Rev. C 77, 065201 (2008).

[69] V. V. Braguta and A. Y. Kotov, Phys. Rev. D 93, 105025 (2016).

[70] R. L. S. Farias, D. C. Duarte, G. Krein, and R. O. Ramos, Phys. Rev. D 94, 074011 (2016).
[71] D. C. Duarte, R. L. S. Farias, and R. O. Ramos, Phys. Rev. D 99, 016005 (2019).

[72] T. Graf, J. Schaffner-Bielich, and E. S. Fraga, Phys. Rev. D 93, 085030 (2016).

[73] S. P. Klevansky, Rev. Mod. Phys. 64, 649 (1992); T. Hatsuda and T. Kunihiro, Phys. Rep. 247, 221 (1994); M. Buballa, Phys. Rep. 407, 205 (2005); H. Kohyama, D. Kimura, and T. Inagaki, Nucl. Phys. B906, 524 (2016).

[74] P. Rehberg, S. P. Klevansky, and J. Hufner, Phys. Rev. C 53, 410 (1996).

[75] R. Câmara Pereira, J. Moreira, and P. Costa, Eur. Phys. J. A 56, 214 (2020); P. Costa and R. C. Pereira, Symmetry 11, 507 (2019). 